\title{
Electro-Polishing Foil Samples for TEM with an Extremely Small Amount of Electrolyte
}

\author{
H.Saka $^{1}$, M.Yamamoto $^{2}$, Y.Shiraishi $^{2}$, M.Amano $^{2}$, T.Goto $^{2}$, T.Suzuki $^{3}$, S.Arai $^{3}$ and K.Sasaki ${ }^{1}$ \\ ${ }^{1}$ Nagoya University, School of Engineering, Nagoya, Japan \\ 2.Yamamoto Chemical Co, Hiroshima, Japan \\ ${ }^{3 .}$ Nagoya University, Ecotopia Science Institute, Nagoya, Japan
}

An innovative method to electro-polish metallic material to electron transparency, where only an extremely small amount of electrolyte (less than a few $\mathrm{ml}$ ) is used, is developed and applied to a few metallic materials.

The principle of the method is illustrated in Fig.1. A conventional $3 \mathrm{~mm} \varphi$ disc is sandwiched between a pair of ring foils ( $\mathbf{1}$ and $\mathbf{2} ; 0.15 \mathrm{~mm}$ thick, stainless steel), together with a spacer $(\mathbf{3} ; 0.15 \mathrm{~mm}$ thick, stainless steel). This assembly is mounted into an electro-polishing cell (Fig.2), which is in turn mounted to the electro-polisher.

The electro-polishing cell is connected to the anode via a guide $\mathbf{G}$ and a needle cathode is lowered, the distance between the tip of the cathode and the sample being adjusted by observing under optical microscope [1]. Electrolyte is poured with a pipette and electro-polishing starts.

The lower surface of the sample is monitored by an optical microscope [2]. The first step is to electropolish the upper surface of the sample, which is interrupted well before a hole is penetrated. Then the cell is turned over and the other (bottom) surface is polished while the now polished upper surface is being observed by an optical microscope [3] and displayed on a TV screen (Fig.3). The polishing is finalized when a small hole is perforated in the center: The sample is dismounted and cleaned in a usual manner.

Fig.4a shows a DF image of 17-7PH steel taken with a forbidden spot shown in the diffraction pattern. Fig.4b shows a HREM of the precipitate in Fig.4. The precipitate shows lattice twice as large as the matrix. Fig.5 shows EDX analysis; the precipitates are identified as $\beta$ '-NiAl in good agreement with [1]. Fig.6 shows precipitate of $\mathrm{Ni}_{3} \mathrm{Al}$ in a Ni-base super-alloy. These micrographs were taken in a $\mathrm{Cs}$ corrected microscope ARM operated at $200 \mathrm{kV}$.

\section{References:}

[1] N Yukawa, M Mizutani and H Saka, $6^{\text {th }}$ International Congress for Electron Microscopy, Kyoto(1966) p.403.

[2] This work was carried out in cooperation with Excellent Support Center for Reaction, Nanomaterials and Biological Science by Electron Microscopy, Nagoya University.

[3] H. S thanks the Ministry of Education, Culture, Sports, Science and Technology for the financial support (Grant-in-Aid for Scientific Research (C), Contract \#24560803). 


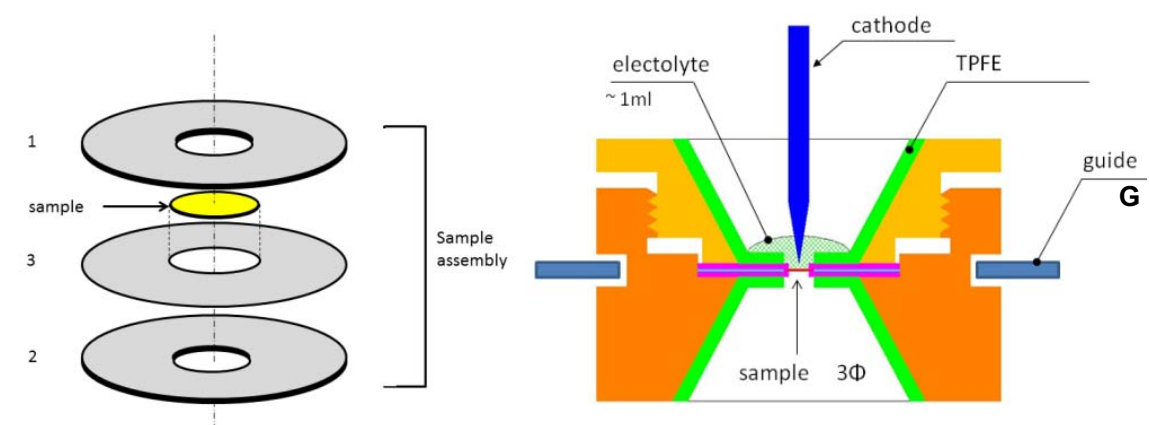

Figure 1. Sample assembly. Figure 2. Electro-polishing cell.

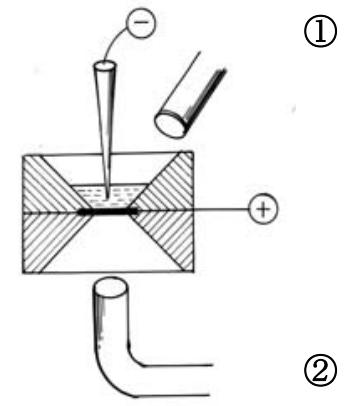

Figure 3. Monitoring

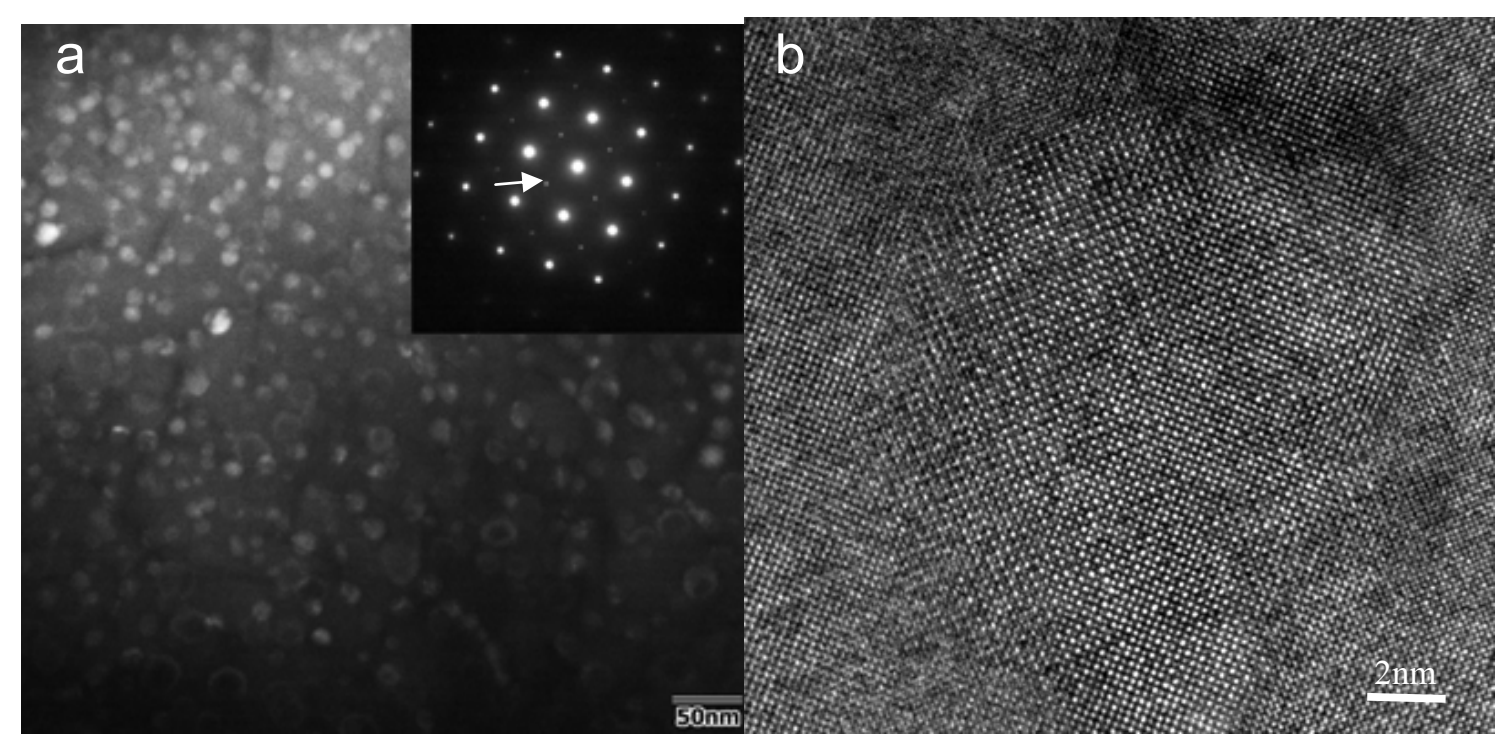

Figure 4. (a) DF image of 17-7PH stainless steel taken with a forbidden spot (indicated by arrow) in the diffraction pattern (inset). (b) HREM of a precipitate.

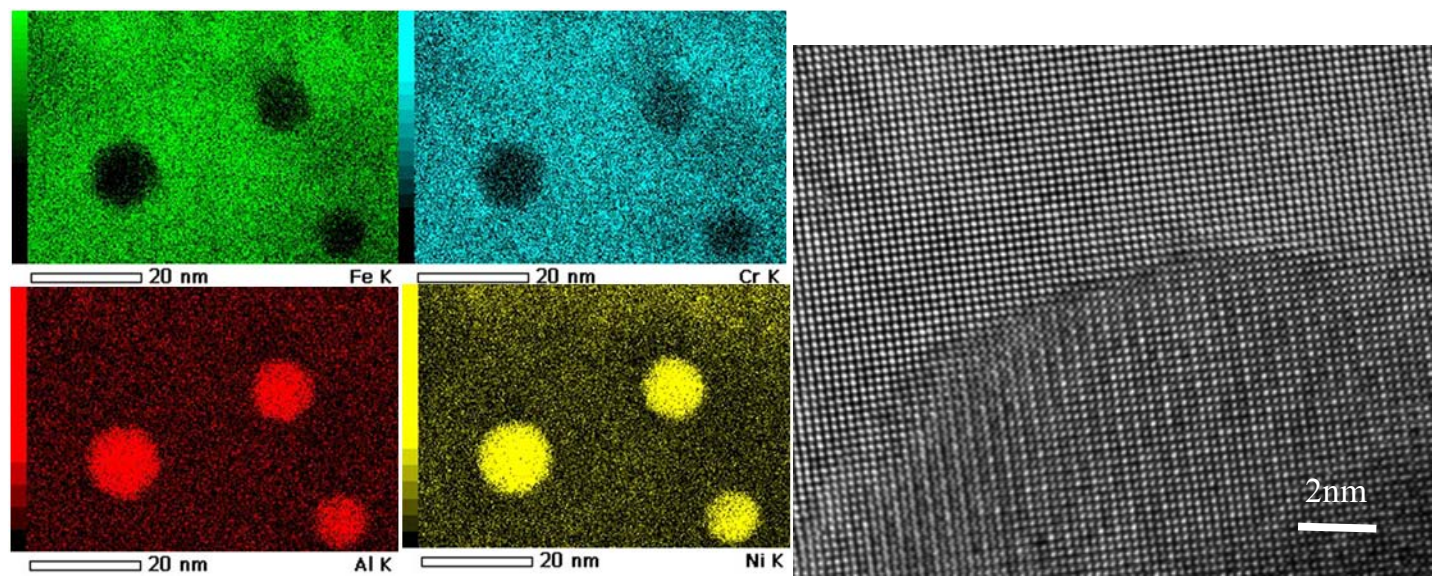

Figure 5. EDX mapping

Figure 6. HREM of $\gamma$ ' in Ni-Al. 